\title{
Difference of Predicted Values by Near-infrared Spectrometers Caused by Wavelength Resolution
}

\author{
Tsutomu OKURA*, Shanji PIAO* and Sumio KAWANO** \\ *Research \& Development Division, SOMA OPTICS, LTD. \\ **Faculty of Agriculture, Kagoshima University
}

Received March 6, 2014, Accepted September 29, 2014

\begin{abstract}
Near-infrared spectroscopy (NIRS) is an efficient and non-destructive method for quantitatively analyzing ingredients in a material. However, this method requires an expensive and time-consuming process to establish a calibration model. As a consequence, once the calibration model is established, it usually is used for many other spectrometers. Then, more or less a disparity in the predicted ingredient content of the material is observed. This phenomenon is called the instrumental difference and gives a difficulty in the application of NIRS. The reason is that waveform of near-infrared (NIR) spectrum is influenced by the characteristics of the spectrometer. We examined the process of measurement and found the spectral waveform was distorted by the variation of spectral response within wavelength resolution of the spectrometer. Therefore, the large spectral response variation and low wavelength resolution causes the big waveform distortion and then the big difference of predicted value of contents. Using seven linear array spectrometers, we analyzed this distortion and evaluated the difference of the predicted value. The results coincided with the analysis. By understanding the mechanisms of instrumental difference, we can design a spectrometer with minimal instrumental difference. This result could be applied to any other spectroscopy that acquires spectral waveform by dividing the sample waveform by the reference waveform.
\end{abstract}

KEYWORDS: near-infrared spectroscopy, chemometrics, wavelength resolution, spectroscopy, calibration model

\section{Introduction}

Quantitative analysis using reflectance or transmittance spectra in the near-infrared (NIR) wavelength region was enabled by the research of Karl Norris during the late 1960s and $1970 \mathrm{~s}^{122}$. The method is non-destructive and relatively easy to use, and so has been applied in many fields such as food, pharmacy, agriculture, stock farm products, and industrial products ${ }^{3}$. In order to retrieve information from NIR spectra which is dull without any sharp peaks, calibration models by statistics are necessary for the application of NIRS quantitative analysis ${ }^{1}$. Many samples need to be gathered to generate a calibration model. These calibration samples must then be chemically analyzed to measure the content of the ingredient. Then, the relationship between the chemically analyzed ingredient content and the measured NIR spectra are analyzed using chemometrics to yield a calibration model. Using this calibration model, prediction of ingredient content of sample is possible. Compared to time-consuming and tedious chemical analysis requiring the application of reagents, NIRS is fast and easy method. Quantitative analysis by NIRS can be used instead of chemical analysis at sites requir- ing rapid analysis ${ }^{4)}$.

Each of the steps of the development of calibration models for NIRS is costly and time-consuming. In addition, the calibration model must be revised when the optical characteristics of spectrometers or samples vary over time. Once a calibration model is established, it is usually applied to many spectrometers. However, the predicted content values acquired by different spectrometers using the same calibration model do not always coincide. This phenomenon is called instrumental difference. Each NIR spectrometer usually has its own small spectral shape difference coming from optical characteristics of the hardware. In NIRS, even the subtle difference of the spectral shape is used to predict the ingredient content. So, instrumental difference is likely to occur.

Many methods called standardization are being used to minimize instrumental differences and facilitate the transfer of calibration models across different spectrometers ${ }^{5)}$, such as adjusting slope and bias of predicted values $^{6)}$, compensating waveform of NIR spectra ${ }^{78)}$ and others ${ }^{910)}$. These methods do not intend to eliminate the causes behind instrumental difference but instead, ad- 
just the resulting predicted values or spectra. Such methods would sometimes degrade the performance of predictions.

Investigating mechanisms of instrumental difference is crucial for the development of NIRS. We analyzed the process how difference of NIR spectra was derived from measurements. In this analysis, it turned out that wavelength resolution of the spectrometer played an important role.

The relation between wavelength resolution and spectral shape had been investigated since the grating spectrometer was developed ${ }^{1112)}$. On the basis of these analysis, the relation between the waveform of the infrared absorption and wavelength resolution is finely discussed ${ }^{1314)}$. This relation was also discussed for spectral illumination measurement in order to get accurate integrated value ${ }^{15}$. However, those analyses had never taken account of the spectral response of the spectrometer which is the efficiency of the spectrometer at each wavelength.

In our analysis, we took account of the spectral response and found that the differences came from variation of the spectral response within wavelength resolution and it became bigger by the lower wavelength resolution.

The spectral response $H(\lambda)$ of a spectrometer at wavelength $\lambda$, was defined here as the signal intensity spectrum when an imaginary 100\% reflectance sample was measured by a spectrometer and was influenced by the light source spectral distribution, reflectance of gratings and mirrors, transmittance of lenses, and spectral sensitivity of detectors used in spectrometers. Reflectance of gratings ${ }^{16)}$ and spectral sensitivities of detectors ${ }^{17)}$ vary greatly and are the main causes of the difference of spectral response of spectrometers.

We examined the result of our analysis by measuring samples and predicting the ingredient content using seven spectrometers with the same design. However they were with the same design, the spectrometers had different spectral responses because of the spectral sensitivity of linear array detectors. Therefore, the predicted values by different spectrometers had considerable difference. The relation between spectral response and the instrumental difference coincided with our analysis.

We analyzed the role of spectral response of the spectrometer in the instrumental difference. We found the relation between the spectral response and the instrumental difference. It was to be expected that bigger differences in the spectral response would yield bigger instrumental difference and this phenomenon became bigger under the lower wavelength resolution. Knowing the reason, it will become possible to decrease or avoid the instrumental difference.

\section{Theoretical analysis}

Our analysis was based on grating-type spectrometers, but the theory applies equally to other spectrometers with finite wavelength resolution. Although we considered only reflectance spectra for simplicity of the analysis, the results are valid for transmittance spectra as well.

\subsection{Measurement of spectra by spectrometers}

In NIRS, the measured reflectance spectrum $R(\lambda)$ is the ratio between the intensity of the light reflected by the sample and the white reference, multiplied by the reflectance of the white reference at each wavelength ${ }^{18)}$.

$$
R(\lambda)=\frac{x(\lambda)}{w(\lambda)} \cdot W(\lambda)
$$

where

$\lambda$ is the wavelength,

$R(\lambda)$ is the measured reflectance spectrum,

$x(\lambda)$ is the measured intensity spectrum of sample,

$w(\lambda)$ is the measured intensity spectrum of the white reference, and

$W(\lambda)$ is the reflectance spectrum of the white reference.

The white reference usually has a high reflectance spectrum close to $100 \%{ }^{19}$. The influence of the spectral response on the measured intensity spectrum is expected to be compensated by dividing the sample intensity by the white reference intensity as shown by Equation (1). However, by our analysis, this expectation turned out to be not valid.

\subsection{Influence of spectral response}

According to former analyses ${ }^{12)-14)}$ on spectral shape measured by a spectrometer, the relation between the measured reflectance spectrum $R(\lambda)$ (Equation (1)) and the true reflectance spectrum $X(\lambda)$ of the sample is expressed by a convolution integral (Equation (2)) using the slit function $\Delta(a)$ of the spectrometer. Variable $a$ is wavelength shift from wavelength $\lambda_{0}$. The integration limits $\pm D$ is the area where $\Delta(a)$ is not zero. The slit function is considered to be triangular or trapezoid shape according to the entrance and exit slit width ${ }^{14) 15}$.

$$
R\left(\lambda_{0}\right)=\frac{\int_{-D}^{+D} X\left(\lambda_{0}-a\right) \cdot \Delta(a) \cdot \mathrm{d} a}{\int_{-D}^{+D} \Delta(a) \cdot \mathrm{d} a}
$$

where

$\lambda_{0} \quad$ is wavelength of the spectrometer,

$X\left(\lambda_{0}\right)$ is true reflectance spectrum,

$a \quad$ is wavelength shift from $\lambda_{0}$,

$\Delta(a)$ is slit function of the spectrometer, and

$D \quad$ is integration limit.

Equation (2) is widely acknowledged as the equation 
for the reflectance spectra ${ }^{13)}$. In the Equation (2), the influence of the spectral response $H(\lambda)$ on the measured spectrum $R(\lambda)$ is neglected. Therefore, according to Equation (2), all spectrometers with the same slit function $\Delta(a)$ would produce the same reflectance spectra. Nevertheless, the reflectance spectra measured by different spectrometers show differences and this is caused by the difference between individual spectrometer's spectral response.

Considering the effect of spectral response of the spectrometer $H(\lambda)$ on reflectance spectra, the signal intensity spectrum of the sample $\mathrm{x}(\lambda)$ and white reference $w(\lambda)$ measured by the spectrometer are

$$
\begin{aligned}
& x\left(\lambda_{0}\right)=\frac{\int_{-D}^{+D} X\left(\lambda_{0}-a\right) \cdot H\left(\lambda_{0}-a\right) \cdot \Delta(a) \cdot \mathrm{d} a}{\int_{-D}^{+D} \cdot \Delta(a) \cdot \mathrm{d} a} \\
& w\left(\lambda_{0}\right)=\frac{\int_{-D}^{+D} W\left(\lambda_{0}-a\right) \cdot H\left(\lambda_{0}-a\right) \cdot \Delta(a) \cdot \mathrm{d} a}{\int_{-D}^{+D} \Delta(a) \cdot \mathrm{d} a}
\end{aligned}
$$

where

$H(\lambda)$ is spectral response of the spectrometer.

The slit function $\Delta(a)$ in the Equations (3) and (4) is defined not only by the optics of the spectrometer but also by the data smoothing of the measured intensity spectrum. Combining Equations (1), (3), and (4), the measured reflectance spectrum was given by

$$
\begin{aligned}
R\left(\lambda_{0}\right) & =\frac{x\left(\lambda_{0}\right)}{w\left(\lambda_{0}\right)} \cdot W\left(\lambda_{0}\right) \\
& =\frac{\int_{-D}^{+D} X\left(\lambda_{0}-a\right) \cdot H\left(\lambda_{0}-a\right) \cdot \Delta(a) \cdot \mathrm{d} a}{\int_{-D}^{+D} W\left(\lambda_{0}-a\right) \cdot H\left(\lambda_{0}-a\right) \cdot \Delta(a) \cdot \mathrm{d} a} \cdot W\left(\lambda_{0}\right)
\end{aligned}
$$

The white reference used for NIR spectrometers has high reflectance spectrum $W(\lambda)$ which is considered to be constant within the integration limits (i.e. $W\left(\lambda_{0}-a\right)$ $\left.=W\left(\lambda_{0}\right)\right)$. Equation (5) then became

$$
R\left(\lambda_{0}\right)=\frac{\int_{-D}^{+D} X\left(\lambda_{0}-a\right) \cdot H\left(\lambda_{0}-a\right) \cdot \Delta(a) \cdot \mathrm{d} a}{\int_{-D}^{+D} H\left(\lambda_{0}-a\right) \cdot \Delta(a) \cdot \mathrm{d} a}
$$

and incorporated the influence of $H(\lambda)$ on the measured reflectance spectrum.

\subsection{Instrumental difference}

The Equation (6) told that measured reflectance spectra were influenced by the spectral response of the spectrometer. However, there were two cases in which $R(\lambda)$ was independent of $H(\lambda)$. First, when $H(\lambda)$ was constant (i.e. $H\left(\lambda_{0}-a\right)=H\left(\lambda_{0}\right)=H$ ) within the integration limits, Equation (6) was simplified as

$$
\begin{aligned}
R\left(\lambda_{0}\right) & =\frac{\int_{-D}^{+D} X\left(\lambda_{0}-a\right) \cdot H \cdot \Delta(a) \cdot \mathrm{d} a}{\int_{-D}^{+D} H \cdot \Delta(a) \cdot \mathrm{d} a} \\
& =\frac{\int_{-D}^{+D} X\left(\lambda_{0}-a\right) \cdot \Delta(a) \cdot \mathrm{d} a}{\int_{-D}^{+D} \Delta(a) \cdot \mathrm{d} a}
\end{aligned}
$$

which was the same as Equation (2). Second, when $X(\lambda)$ remained constant (i.e. $\left.X\left(\lambda_{0}-a\right)=X\left(\lambda_{0}\right)\right)$ within the integration limits, Equation (6) became

$$
R\left(\lambda_{0}\right)=\frac{\int_{-D}^{+D} X\left(\lambda_{0}\right) \cdot H\left(\lambda_{0}-a\right) \cdot \Delta(a) \cdot \mathrm{d} a}{\int_{-D}^{+D} H\left(\lambda_{0}-a\right) \cdot \Delta(a) \cdot \mathrm{d} a}=X\left(\lambda_{0}\right)
$$

These two cases above show that when the variation of spectral response or reflectance spectrum of sample in the integration limits was small, the instrumental difference would be small. These cases are likely to happen when the wavelength resolution is high.

For the case of UV-VIS or mid-infrared spectroscopy, where the wavelength resolution is narrower than the half band width of the absorption bands of the sample, Equation (7) or (8) can apply, and the impact of the instrumental difference is reduced.

For the case of NIRS, however, where lower wavelength resolution is preferred to avoid noise, it is likely to confront the instrumental difference which will cause substantial difference in the predicted values.

\subsection{Graphical explanation}

Figure 1 shows the effects of spectral responses. For simplicity, we assume the slit function is rectangular. A spectrometer detects equally all the light intensity within its wavelength resolution. Therefore, the measured value becomes the integral value within the wavelength resolution.

The true reflectance spectrum of a sample is shown at the top of Figure 1. Assume three spectrometers A, $\mathrm{B}$, and $\mathrm{C}$ have different spectral responses and the reflectance spectrum of the white reference is $100 \%$. The reference signal intensities $A_{r}, B_{r}$, and $C_{r}$ are the integrals of the spectral response. We assume that each integrated signal intensities of white reference are the same $\left(A_{r}=B_{r}=C_{r}\right)$.

Then, the sample signal intensities, i.e. $A_{s}, B_{s}$, and $C_{s}$, are shown as shaded areas determined by the product of the sample reflectance spectrum and spectral response of spectrometers. The reflectance spectrum values $R_{A}, R_{B}$, and $R_{C}$ measured by three spectrometers are shown in the lowest position in Figure 1. They are calculated from the intensities of the sample and reference, i.e. $A_{s} / A_{r}, B_{s} / B_{r}$ and $C_{s} / C_{r}$ Figure 1 makes clear that the signal intensities for three cases are different 


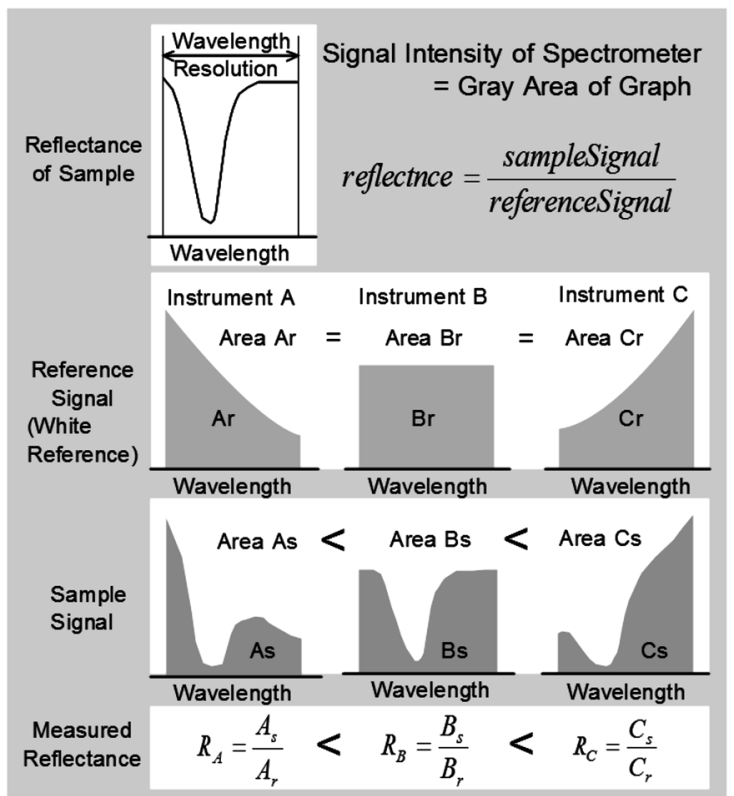

Figure 1 Instrumental difference caused by the spectral response.

$\left(A_{S}<B_{S}<C_{S}\right)$. Therefore, the reflectance spectrum value are different $\left(R_{A}<R_{B}<R_{C}\right)$. This means that the measured reflectance spectrum values differ according to the spectral responses of the spectrometers.

\subsection{Results of the theoretical analysis}

Measurements under the following conditions would result in smaller spectral distortion and smaller instrumental differences.

(a) Small wavelength dependence for the true reflectance spectrum as explained by Equation (8), and

(b) Small wavelength dependence for the spectral response, as explained by Equation (7)

(c) High wavelength resolution (of spectrometer's optical design and data smoothing of intensity spectra).

Issue (a) depends on sample characteristics. Issue (b) is realized by selecting optical parts with flat wavelength characteristics. The wavelength dependence in issue (a) and (b) are evaluated only within the integration limits determined by the wavelength resolution. So, high wavelength resolution of issue (c) would give the narrow integration limits and the wavelength dependence would be small. However, the narrower slit width will give a weak signal and it will be necessary to increase the signal intensity to realize the high SN ratio. The high wavelength resolution needs high wavelength accuracy since the calibration model becomes more sensitive to the wavelength. If the measured reflectance $R(\lambda)$ is convoluted to be lower wavelength resolution after the calculation of reflectance (Equation (1)), the required wavelength accuracy for the spectrometer does not need to be such high.

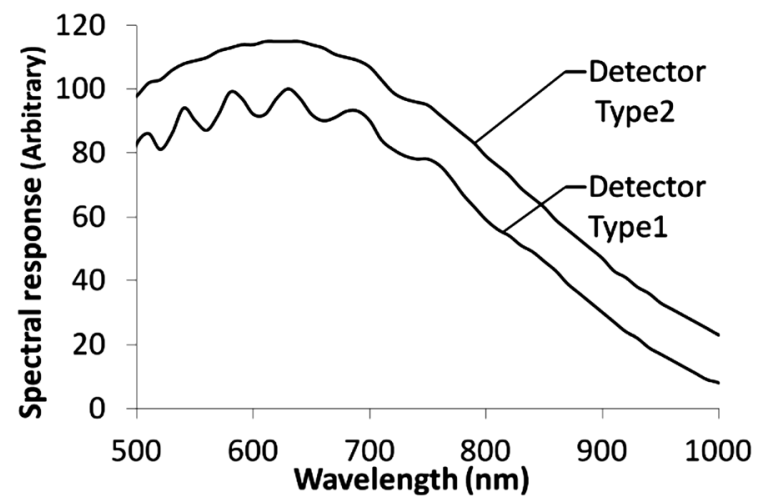

Figure 2 Spectral sensitivity of detectors. Detector type1 exhibits periodic variation. Detector type2 was improved to minimize periodic variation. These data are provided by Hamamatsu Photonics K. $\mathrm{K}^{17}$.

Table 1 Configuration of seven spectrometers

\begin{tabular}{ccc}
\hline Spectrometer & Detector & Hardware configuration \\
\hline H01 H02 H03 & type1 & Wavelength 700-1050 nm \\
\cline { 1 - 1 } N01 N02 N03 & type2 & Resolution 7 nm \\
S01 & type2 & Pixel 512 \\
\hline
\end{tabular}

\section{Material and methods for verification}

\subsection{Samples and spectrometers}

To verify the analysis, seven spectrometers of the same design were used to predict the fatty acid composition of meat fat ${ }^{2021)}$. Though the seven spectrometers are the same design, each spectral response cannot be the same because of the spectral sensitivity of the detector.

26 beef meat fat block samples were used as samples for this experiment to predict the oleic fatty acid composition. Fatty acid composition in meat fat is one of the most important indices of meat quality. NIR method is effective for this case since measurement of fatty acid composition by gas chromatography needs tedious procedure and long time around few hours.

One spectrometer ( $\mathrm{S} 01)$ was used as a standard and each of the other six spectrometers in two groups, i.e. (H01, H02, and H03) and (N01, N02, and N03) was compared against (S01). The spectrometers were grating spectrometers with linear array detectors with a wavelength range of 700 to $1050 \mathrm{~nm}$ and wavelength resolution was $7 \mathrm{~nm}$. The linear array detectors were CMOStype silicon linear array with 512 pixels, manufactured by Hamamatsu Photonics K.K ${ }^{17)}$. The spectral sensitivity of the linear array detectors varied periodically according to wavelength (detector type 1 in Figure 2). This periodic variation was due to optical interference in the film structure on the sensing area of the detector and changed with each manufacturing process. The 
amplitude and cyclic wavelength period changed so much that these became the main causes of the spectral response variation of the spectrometers. Recently, this is improved to have smaller periodic variation (detector type 2 in Figure 2). The improved detectors of type 2 were installed to S01, N01, N02, and N03. Detectors of type 1 with large periodic variations were installed to H01, H02, and H03 (Table1).

\subsection{Wavelength resolution and evaluation method}

We evaluated the case for 7 and $14 \mathrm{~nm}$ wavelength resolutions which represented high and low wavelength resolution. Evaluation of the instrumental difference was done by two ways: (a) by the measured spectra by Equation (1), and (b) by the calculated spectra by Equation (6) using a simulated true reflectance spectrum and measured spectral responses of seven spectrometers.

(a) Evaluation of the measured spectra

The measured reflectance spectra were acquired by the Equation (1) using the measured intensity spectra of the samples and the white reference for both of 7 and $14 \mathrm{~nm}$ wavelength resolution and were compared. The white reference was assumed to be $100 \%(W(\lambda)=1)$.

As the wavelength resolution of the spectrometers was $7 \mathrm{~nm}$, the measured intensity spectra were used directly for the $7 \mathrm{~nm}$ wavelength resolution evaluation without any data smoothing. We applied $12 \mathrm{~nm}$ width data smoothing to measured intensity data of $7 \mathrm{~nm}$ wavelength resolution and used the data as $14 \mathrm{~nm}$ wavelength resolution. We had examined that this process yielded $14 \mathrm{~nm}$ wavelength resolution data.

Dividing these sample intensity spectra by white reference intensity spectra i.e. Equation (1), the measured reflectance spectra for 7 and $14 \mathrm{~nm}$ wavelength resolution were acquired.

After the calculation of Equation (1), data smoothing was applied to be the same wavelength resolution of $16.3 \mathrm{~nm}$ to compare 7 and $14 \mathrm{~nm}$ reflectance spectra under the identical conditions. 14 and $7 \mathrm{~nm}$ width data smoothing were applied to 7 and $14 \mathrm{~nm}$ wavelength resolution reflectance spectra, respectively. The data smoothing of acquired reflectance spectra would not give any more instrumental difference. Then reflectance spectra were transformed into absorbance and second order derivatives were calculated by Savitzky \& Golay-method ${ }^{22)}$.

For both of 7 and $14 \mathrm{~nm}$ evaluations, the second order derivative spectra for 26 samples by seven spectrometers were averaged for each spectrometer. Applying the calibration model of oleic fatty acid composition to these averaged second order derivative spectra, we evaluated how the predicted values of each spectrometer differed from the standard spectrometer (S01). The predicted values by six spectrometers (N01, N02, N03, $\mathrm{H} 01, \mathrm{H} 02$, and H03) were subtracted by the predicted value of standard spectrometer (S01).

The calibration model used here for the oleic fatty acid composition had been previously established by 833 beef meat fat samples ${ }^{23}$. This calibration model had the correlation factor 0.83 and the standard error of the calibration $1.91 \%$ while the fatty acid composition of the oleic acid ranged from 43 to $65 \%$.

(b) Evaluation of the calculated spectra

The reflectance spectra were calculated by the Equation (6), assuming that (1) the average of all the 182 measured reflectance spectra (26 samples measured by the 7 spectrometers) was the true reflectance spectrum of the sample $X(\lambda),(2)$ measured intensity spectra of the white reference by seven spectrometers were the spectral responses $H(\lambda)$ and (3) the slit function of 7 and 14 nm were triangular shape.

By this evaluation, the instrumental difference could be estimated without being influenced by the hardware of the spectrometer (i.e. wavelength accuracy, noise, and sample optics) other than spectral response.

After the calculation of Equation (6), the same data processing as in evaluation (a) were applied.

\section{Results and discussion}

The spectral responses (intensity spectra of white reference) of all the spectrometers ( $\mathrm{H} 01, \mathrm{H} 02, \mathrm{H} 03, \mathrm{~N} 01$, N02, N03, and S01) and the sample reflectance spectrum (in abs) used in the evaluation are shown in Figure 3. Each spectrum is shifted upwards as a visual aid.

The spectral responses for $\mathrm{H} 01, \mathrm{H} 02$, and $\mathrm{H} 03$ with the detectors type 1 showed large periodic variations while S01, N01, N02, and N03 with the detectors type 2 showed smaller periodic variations.

The differences of the second order derivative spec-

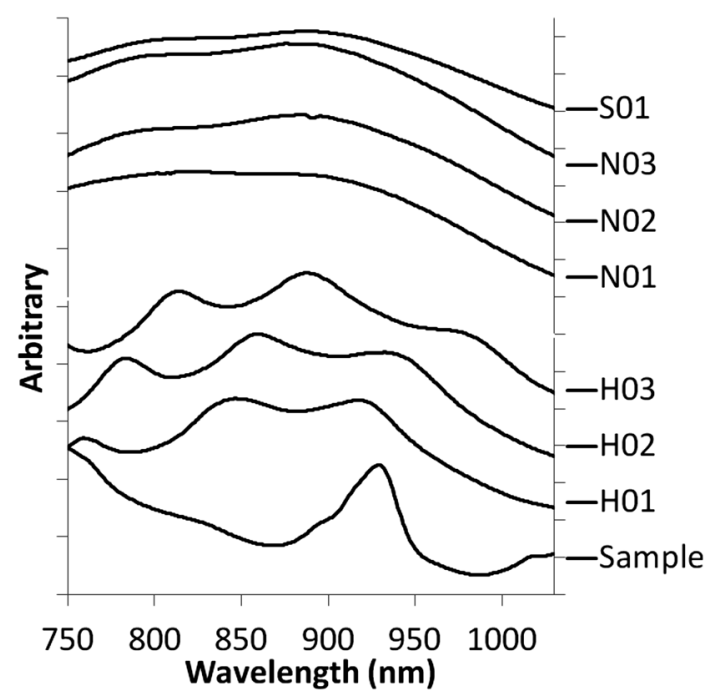

Figure 3 Spectral responses of the seven spectrometers ( H01, H02, H03, N01, N02, N03 and S01) and averaged absorbance of the samples. 

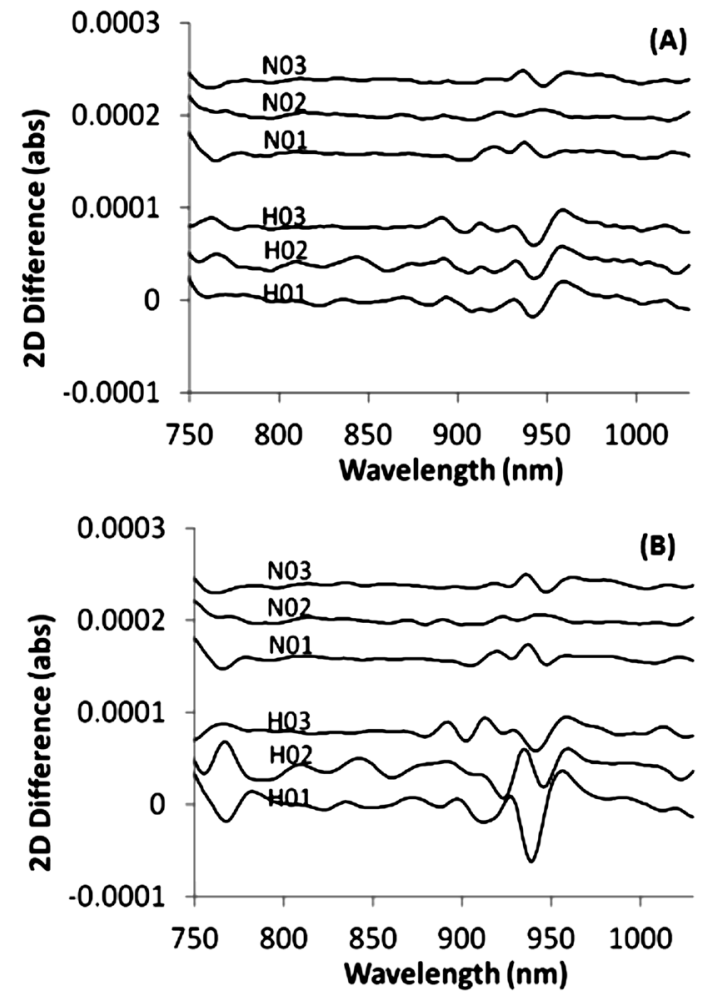

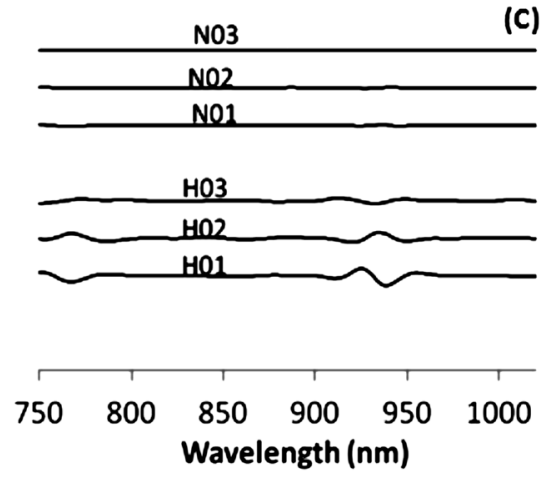

(D)

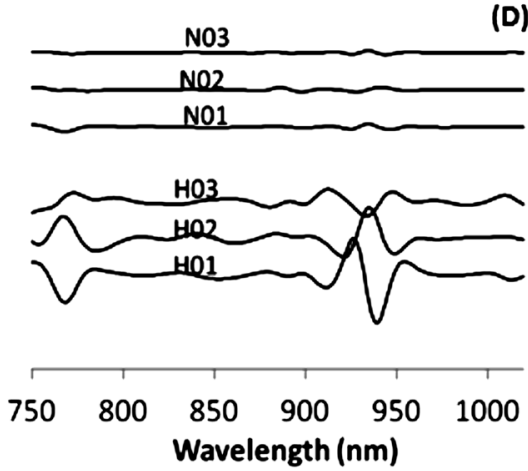

Figure 4 Differences of the second order derivative spectra of the six spectrometers (H01, H02, H03, N01, N02, N03) against (S01).

(A) measured spectra (7 nm wavelength resolution), (B) measured spectra (14 $\mathrm{nm}$ wavelength resolution), (C) calculated spectra (7 $\mathrm{nm}$ wavelength resolution), (D) calculated spectra (14 $\mathrm{nm}$ wavelength resolution).

tra for (a) measured spectra and (b) calculated spectra against the standard spectrometer S01 are shown in Figure 4.

All the spectra had bigger spectral differences around $930 \mathrm{~nm}$ which was the peak of fat absorption. This phenomenon coincided with (a) in paragraph 2.5.

All the spectra by N01, N02, and N03 had smaller differences. This was because of smaller spectral response variance of the spectrometer. This phenomenon coincided with (b) in paragraph 2.5.

All the spectra in (A) and (C) for higher resolution (7 $\mathrm{nm}$ ) showed lower differences. This coincided with (c) in paragraph 2.5.

Tendency of the differences of the measured spectra in (A) and (B) were the same as the calculated spectra (C) and (D) which told us that our analysis was correct. However, (A) and (B) had bigger differences. This was because of the wavelength accuracy of the spectrometers. When we used simulated true spectrum as in the case of $(C)$ and $(D)$, there were no wavelength differences. Therefore, the differences caused by the wavelength accuracy were not observed.

Results were as follows.

(1) Differences were larger in the wavelength area around $930 \mathrm{~nm}$ where the wavelength dependence of the reflectance spectrum was large.
(2) Spectrometers with the detectors of large wavelength dependence $\mathrm{H} 01, \mathrm{H} 02$ and $\mathrm{H} 03$ exhibited larger differences compared to the spectrometers with the detectors of small wavelength dependence N01, N02, and N03.

(3) Higher wavelength resolution $7 \mathrm{~nm}(\mathrm{~A})$ and (C) exhibited smaller differences than lower wavelength resolution $14 \mathrm{~nm}(\mathrm{~B})$ and (D).

These results coincided with (a), (b), and (c) in paragraph 2.5 and verified our analysis was valid.

The differences of the second order derivatives in Figure 4 were evaluated by applying the calibration model of oleic fatty acid composition and are shown in Figure 5. Figure 5 exhibits the difference of the predicted values of oleic fatty acid composition in meat fat against the standard spectrometer S01.

When the differences of the second order derivative spectra were large (H01, H02, and H03 in Figure 4 (B) and (D)), the differences of the predicted value were big (H01, H02, and H03 in Figure 5 (B) and (D)) and reached $10 \%$, while the standard error of the calibration model was $1.91 \%$. Therefore, appropriate standardization was necessary.

The higher wavelength resolution with narrower slit width and/or smaller data smoothing width gave smaller instrumental differences in the predicted values (N01, 

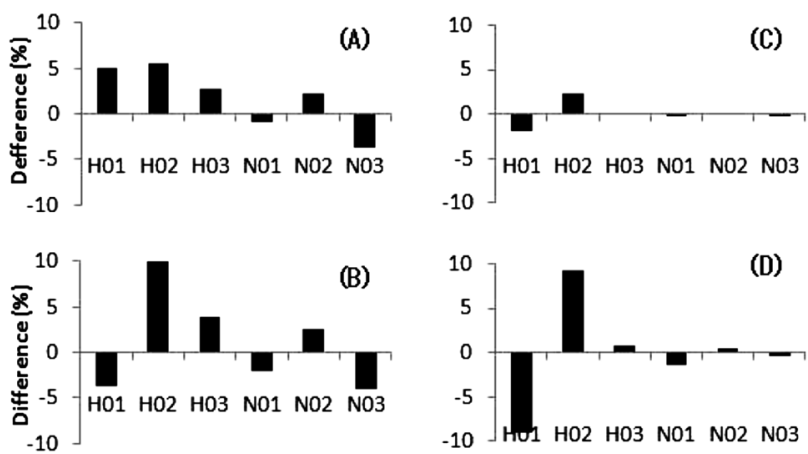

Figure 5 Differences of the predicted value against the Standard Spectrometer S01.

(A) measured spectra (7 $\mathrm{nm}$ wavelength resolution), (B) measured spectra (14 $\mathrm{nm}$ wavelength resolution), (C) calculated spectra (7 $\mathrm{nm}$ wavelength resolution), (D) calculated spectra (14 $\mathrm{nm}$ wavelength resolution).

N02, and N03 in Figure 5 (A) and (C)). However, the higher wavelength resolution would cause the larger noise and degrade the prediction performance. For the case of the prediction of oleic fatty acid composition, in order to realize effective prediction, noise should be less than $50 \mu \mathrm{abs}^{20121}$. Balancing these factors (wavelength resolution and noise) is crucial when designing an NIR spectrometer.

\section{Conclusion}

We have shown how the wavelength resolution of spectrometers generated instrumental differences and evaluated these effects using seven spectrometers and 26 samples with an established calibration model. We concluded that instrumental differences were greater with big variation of the reflectance spectrum and the spectral response within the wavelength resolution.

Knowing this may facilitate the effective standardization of the spectrometers and the design of more precise spectrometers with less instrumental difference.

It would be possible in future to find the way to avoid the spectral distortion of the spectrometer by correcting the spectral waveform using the spectral response and measured spectral waveform. This correction would make the instrumental difference smaller and the standardization would be more applicable.

\section{Acknowledgement}

We sincerely appreciate Professor Irie at Miyazaki University, who taught us how to perform the spectral measurement and chemical analysis of the fatty acid compositions of meat fat, as well as Hamamatsu Photonics K. K., which provided information pertaining to the characteristics of the linear array detector and improved these characteristics for us.

\section{References}

(1) Ben-Gera, I. and Norris, K. H.: Direct spectrophotometric determination of fat and moisture in meat products, J. Food Sci., Vol. 33(1), pp. 64-67 (1968).

(2) Watson, C.: Near infrared refelctance spectrophotometric analysis of agricultural products, Analytical Chemistry, Vol. 49(9), pp. 835-840 (1977).

(3) Hindle, P.: Historical Development, Handbook of NIR Analysis Third Edition: Ed. by Burns D. and Ciurczak, E., CRC Press, U.S.A., pp. 3-6 (2007).

(4) Blanco, M. and Villarroya, I.: NIR spectroscopy: a rapid-response analytical tool trends in analytical chemistry, Trends in Analytical Chemistry, Vol. 21(4), pp. 240-250 (2002).

(5) Bouveresse, E. and Campbell, B.: Transfer of Multivariate Calibration Models Based on Near-Infrared Spectroscopy, Handbook of NIR Analysis Third Edition: Ed. by Burns, D. and Ciurczak, E. CRC Press, FL33487, U.S.A., pp. 231-243 (2007).

(6) Osborne, B. G. and Fearn, T. J.: Collaborative evaluation of universal calibrations for the measurement of protein and moisture in flour by near infrared reflectance, International Journal of Food Science \& Technology, Vol. 18, pp. 453-460 (1983).

(7) Shenk, J. S. and Westerhaus, M. O.: U.S. Patent No. 4866644 (1991).

(8) Bouveresse, E. and Massart, D. L.: Improvement of the piecewise direct standardization procedure for the transfer of NIR spectra for multivariate calibration, Chemometrics and Intelligent Laboratory Systems, Vol. 32, pp. 201-213 (1996).

(9) Forina, M. et al.: Transfer of calibration function in NIR spectroscopy, Chemometrics and Intelligent Laboratory Systems, Vol. 27(2), pp. 189-203 (1995).

(10) Walczak, B., Bouveresse, D. L., and Massart, D. L.: Standardization of near-infrared spectra in the wavelet domain, Chemometrics and Intelligent Laboratory Systems, Vol. 36, pp. 41-51 (1997).

(11) Brodersen, S.: Slit-width effects, J. O. S. A., Vol. 44(1), pp. 22-25 (1954).

(12) Kudoh, K.: Basis and Method of Spectroscopy, OHM, Tokyo, pp. 423-430 (1985).

(13) Ramsey, D. A.: Intensities and shapes of infrared absorption bands of substances in the liquid phase, J. Am. Chem. Soc., Vol. 74, pp. 72-80 (1952).

(14) Seshadri, K. S. and Jones, R. N.: The shapes and intensities of infrared absorption bands-A review, Spectrochimica Acta, Vol. 10, pp. 1013-1085 (1963).

(15) CIE. 1984.: The Spectroradiometric Measurement of Light Sources, Publiction CIE. No. 63. (1984).

(16) Newport Richardson Grating Catalog. http://www. 
gratinglab.com/Home.aspx Accessed Jul. 04 (2014).

(17) Hamamatsu Photonics. K. K, CCD Catalog. http:// www.hamamatsu.com/jp/ja/product/category/ 3100/index.html Accessed Jul. 05 (2014).

(18) Okura, T. and Hattori, S.: NIR Spectroscopy II. Instrumental technology for NIR spectroscopic measurement, Bunkou Kenkyu, Vol. 53 (2), pp. 109-127 (2004).

(19) Springsteen, A.: Standards for reflectance measurements, parts I, Spectroscopy, Vol. 15(5), pp. 21-27 (2000).

(20) Okura, T., Park, S., Nishioka, T. and Irie, M.: Development of a pork fat evaluation system by NIR spectroscopy, J. Illum. Engng. Inst. Jpn., Vol. 93(8A), pp. 492-500 (2009).

(21) Okura, T., Park, S., Nishioka, T. and Irie, M.: Pork fat evaluation system by near infrared spectroscopy, Proceedings of the 14th International Conference on NIR Spectroscopy, pp. 523-526 (2009).

(22) Savitzky, A. and Golay, M. J. E.: Smoothing and Differentiation of data by simplified least square procedures, Analytical Chemistry, Vol. 36(8), pp. 16271639 (1964).

(23) Okura, T. and Piao, S.: Evaluation of wagyu fatty acid composition by near infrared spectroscopy, Proceedings of the 4th Asian NIR Symposium, pp. 251-252 (2014). 